\title{
ADMINISTRAÇÃO JAPONESA
}

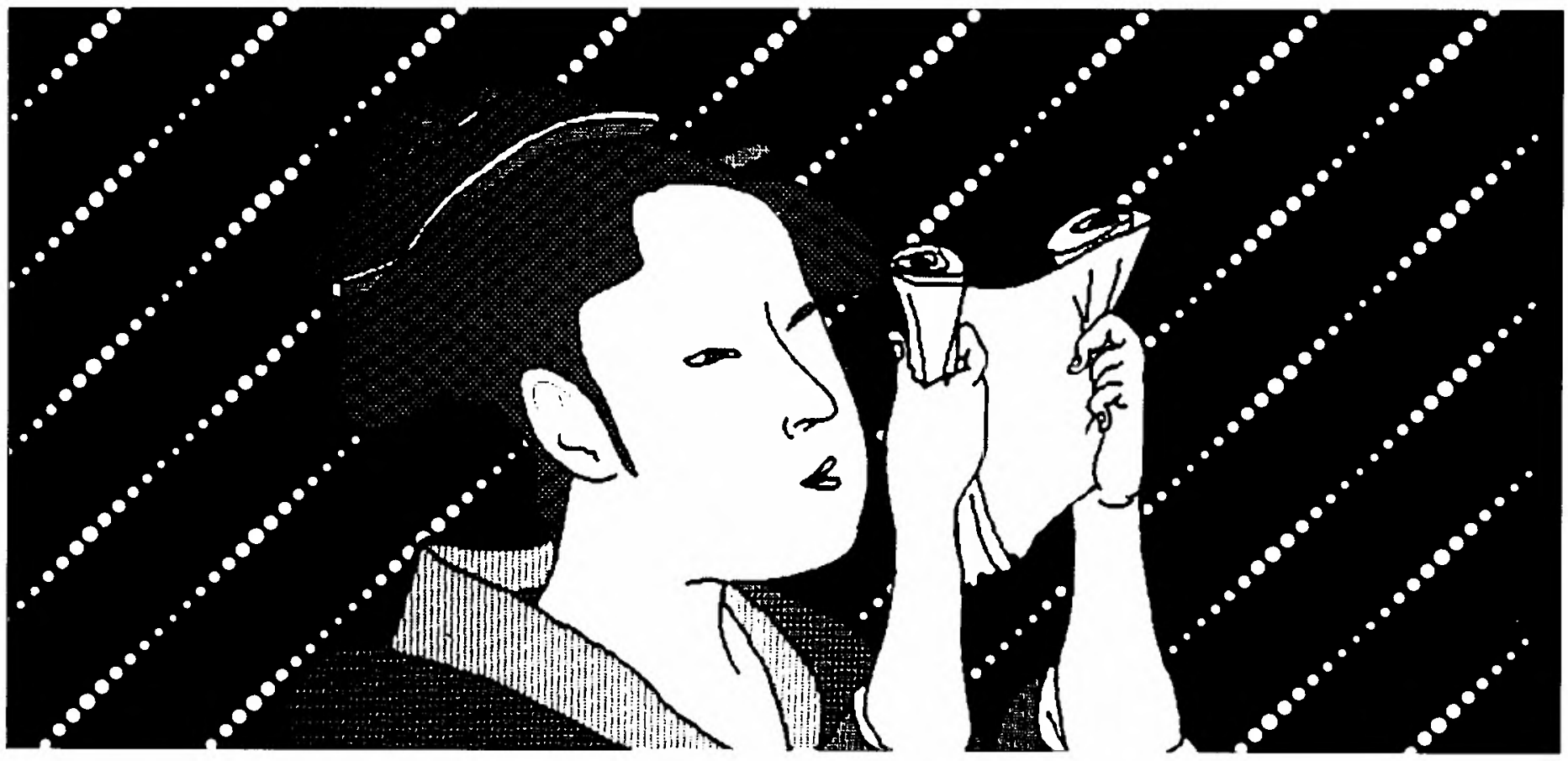

\section{Alberto I. Sugo}

Professor do Departamento de Administração Geral e Recursos Humanos da EAESP/FGV.

\section{APRESENTAÇÃO}

0 interesse na Administração Japonesa, respaldado pelo sucesso japonês, tem sido realimentado constantemente pela publicação de um grande número de artigos e reportagens, formando o que Keys e Miller (ver ref. 077) chamam de "Selva da Teoria Gerencial Japonesa".

Essa renovação constante e variada da literatura impõe a condição de ser necessariamente temporária e genérica qualquer tentativa de levantamento bibliográfico.

No entanto, essa listagem de parte da produção sobre o tema permite a leitura ou o corte adequado para os vários tipos de interessados na Administração Japonesa.

Aberta a possibilidade de se trilhar e abrir qualquer caminho dentro da "selva", sugeriremos, a seguir, um roteiro de leituras.

Tentaremos traçar os contornos gerais do contexto japonês (sua história e cultura), do seu conteúdo (características principais do modelo japonês de gestão) e alguns desenvolvimentos recentes da administração japonesa.

\section{CULTURA E HISTÓRIA DO JAPÃO}

É comum encontrarmos na literatura a idéia de que as práticas gerenciais japonesas são produtos únicos da cul- tura e história japonesa, o que pode levar, com freqüência, a negar a transferência, com sucesso, das receitas gerenciais japonesas para outros países.

Basicamente, sobre essa polêmica temos duas posturas: a da convergência cultural e a do paralelismo cultural.

A primeira entende que as forças da industrialização, ou do processo de acumulação capitalista, levariam necessariamente à mesma forma de gestão capitalista do tipo norte-americano, implicando que formas de gestão ligadas a modos de produção antigos ou pré-capitalistas seriam resquícios de sistemas anacrônicos, que seriam, cedo ou tarde, substituídos.

Essa posição prevaleceu até o início das pesquisas dos grupos ligados a J. Abegglen (ref. 197), mas somente depois de Yoshino (ref. 186) e de Ouchi (ref. 109) perdeu sua força. No entanto, com as tendências de mudanças mais recentes na sociedade japonesa, já identificadas, mas ainda não claramente definidas, podemos ter um retorno modificado e melhorado dessa posição, caso a sociedade, a economia e a gestão japonesas assumam realmente formas já conhecidas do tipo ocidental norte-americano.

A segunda postura entende que as diversas civilizações podem, em virtude das condições locais e históricas, no curso de seu desenvolvimento, encontrar formas alternativas, ou paralelas, para se chegar aos resultados obtidos em outras civilizações. Portanto, não há nenhuma avaliação a priori de que esta ou aquela forma de gestão "paralela" à gestão típica ocidental norte-americana seja inferior ou superior a esta, ou que seja um sinal de atraso tecnoló- 
gico causado por fatores culturais.

Na Antropologia, essa posição sem um etnocentrismo inicial pode ser encontrada nas obras de Lévi-Strauss e nos estudos da antropologia social norte-americana de Ruth Benedict, por exemplo.

Já para a Administração, a posição do paralelismo cultural foi-se fortalecendo graças às obras de Abegglen, Yoshino e Ouchi, permitindo que outros trabalhassem com a idéia de transferência da Administração Japonesa para outros contextos culturais.

Independentemente do pressuposto aceito a respeito da influência da cultura sobre a administração, o conhecimento sobre a história e a cultura japonesas pode servir para uma melhor compreensão do fenômeno administrativo em geral, ao aumentar o leque dos casos e situações disponíveis para análise e comparação.

Em virtude da superficialidade e da facilidade com que alguns autores lançaram conclusões $e$ análises sobre a influência da cultura e história japonesas, torna-se mais necessário o domínio sobre os aspectos culturais e sociais do Japão. Assim, afirmações de que o sistema vitalício de emprego tem raízes centenárias foram corretamente refutadas pela apresentação de antigos e disponíveis estudos históricos, com os quais K. Taira (ref. 194) mostrou que essas práticas são recentes e que se iniciaram depois da rendição do Japão, na Segunda Guerra Mundial. Muitas afirmações levianas têm sido contestadas, mas a literatura, principalmente a de divulgação para executivos, parece ignorá-las e aceitar as crendices com aparência de fatos históricos.

O desconhecimento da língua japonesa e a pouca disponibilidade de material histórico em outras línguas já não podem mais ser desculpa, com o atual movimento de interesse sobre o Japão que tem aumentado a bibliografia disponível, por exemplo, o Japanese Yearbook on Business History (ver refs. 010, 022, 029 e 038) e artigos isolados de Nakaoka (ver refs. 108 e 125), Ozama (refs. 135 e 136), Hazama (ref. 151), Yoshida (ref. 155) e Noguti (ref. 164).

Dois estudos destacam-se na tarefa de apresentar as características fundamentais da sociedade japonesa: $o$ da antropóloga social norte-americana Ruth Benedict (ref. 174) e a visão de "dentro" de Chie Nakane (ref. 058).

$O$ estudo de Benedict, feito a partir de entrevistas com prisioneiros de guerra japoneses nos Estados Unidos, levanta os valores principais dos japoneses e provavelmente ainda não foi superado por nenhuma outra obra na exatidão sobre a compreensão dos sentimentos japoneses.

Nakane aponta a hierarquia e as redes sociais que se interlaçam como elemento caracterizador da sociedade japonesa, em uma análise que lembra o estruturalismo.

Apesar das mudanças mais recentes na sociedade japonesa, esses dois estudos ainda deverão servir como base para a análise das mudanças e do futuro estado da sociedade japonesa.

Podemos citar outros estudos, que, é claro, não pretendem explicar a sociedade japonesa em sua totalidade, mas elucidam aspectos parciais desta: Reischauser (ref. 087), Atsumi (ref. 143), Yamashiro (ref. 192), os painéis de Lebra (ref. 079), Lebra e Lebra (ref. 097), Fodella (ref. 161) e Saito (refs. 137 e 138).

Utilizando-se da pesquisa-participante, o antropólogo
Rohlen (ver refs. 033, 101 e 173) procurou entender as implicações para a gestão do modo de ser japonês, "vivenciando" algumas empresas japonesas: participou dos treinamentos de admissão e estagiários em um banco japonês e de uma empresa de projetos hidráulicos como se fosse um japonês. Para um estudo mais convencional, ver Lauenstein (ref. 053).

\section{MODELOS GERAIS DA ADMINISTRAĢ̃̃O JAPONESA}

As interpretações sobre a Administração Japonesa com maior impacto na literatura são as dos citados Abegglen (ref. 197), Yoshino (ref. 186) e Ouchi (ref. 109). Podemos afirmar que grande parte dos textos sobre a Administração Japonesa copiou, simplificou, mutilou e mentiu, utilizando-se desses textos. Yoshino, por exemplo, fez um estudo sobre a ideologia empresarial japonesa, mas um capítulo de seu livro, sobre a decisão grupal, foi destacado dentro da moda de administração participativa como um modelo a ser seguido, tornando Yoshino um autor da decisão grupal japonesa.

Devemos deixar claro que a bibliografia, na sua maioria, ocupa-se de interpretações sobre o que seria a Administração Japonesa, mas passa sempre a idéia de ser aquele modelo apresentado a própria realidade japonesa, tornando-se um conjunto de mitos ou boatos sobre o Japão. Estudos sem bases empíricas, feitos a partir de viagens turísticas sem nenhum preparo relativo à história e cultura do Japão, são apresentados como a expressão do que realmente acontece no Japão.

Alguns estudos, contudo, são tentativas honestas de sintetizar as características básicas de um modelo da Administração Japonesa, com base em trabalhos empíricos ou em uma reflexão cuidadosa sobre o material existente, objetivando conhecer mais sobre o fenômeno organizacional e sua aplicação a outras situações.

Podemos citar: Pascale e Athos (ref. 112), que vêem a Administração Japonesa como um caso particular dentro do modelo dos Sete Es, contrastando exemplos norte-americanos e japoneses de excelência empresarial; Ishida (ref. 146), que visitou subsidiárias de empresas japonesas no mundo todo; Vogel (ref. 163), que ainda hoje escreve e pesquisa sobre o Japão e suas empresas; Dore (ref. 167), que comparou empresas britânicas com japonesas utilizando um referencial de Burns e Stalker (Administração da Inovação) e Kagono e outros (ref. 121), que, dentro desse mesmo referencial tecnológico, analisam empresas japonesas.

Enquanto modelos que levantam e organizam a bibliografia temos: Dunphy (ref. 028), Negandhi e outros (ref. 059), Keys e Miller (ref. 077), Hatvany e Puick (ref. 118).

O modelo da Administração Japonesa, ao apresentar a possibilidade de haver grande produtividade com a harmonia junto aos trabalhadores, gerou algumas reações críticas ou negativas ao modelo como um todo, ou a alguns de seus elementos, como o do poder nas organizações.

Satoshi (ref. 062), Faria (ref. 070), Odiorne (ref. 084), Sethi e outros (ref. 089), Hirata (ref. 096), Robbins (ref. 100), Tribuna Metalúrgica (ref. 104), Schein (ref. 129) e Ishikawa (ref. 133 e 134). 


\section{DESENOLLVIMENTOS RECENTES}

A forma acidental de ver a Administração Japonesa tem seguido alguns estágios curiosos: um desprezo e arrogância a respeito de primitivos modos de gestão no período pós-guerra, curiosidade no início dos anos 70, imitação frenética no início dos anos 80 , críticas quase xenofóbicas no início dos anos 90 e agora uma assimilação simbiótica ou recuperação de apresentações japonesas como sendo de origem norte-americana. Os estágios japoneses a respeito desse mesmo fenômeno não deixam, tampouco, de ser curiosos.

Assim, atualmente, temos três vertentes principais de desenvolvimentos sobre a Administração Japonesa: uma de crítica e rejeição ao Japão, por exemplo, o ridículo da sopa de letrinhas (CCQ, JIT, KANBAN); uma propondo a assimilação da Administração Japonesa de forma simbiótica e uma, de Japoneses, tentando rever e atualizar o modelo japonês.

A vertente de crítica tenta contrapor às críticas inerentes ao modelo japonês a impossibilidade ou as dificuldades de adaptação do modelo a outros ambientes, e propor uma revitalização dos modelos anteriores, o back to basics, ou o retorno aos valores norte-americanos que teriam feito deste país o líder do mundo, que hoje estaria ameaçado pela acomodação. Essa visão é uma moda que chega também ao Brasil.
A segunda vertente, como podemos notar em vários simpósios-debates promovidos por Harvard, tenta propor modelos que assimilem, na forma de simbiose, tanto valores ou modos norte-americanos como japoneses, e recuperem antigas propostas norte-americanas, como a de Deming e Juran, no contexto de competição internacional e de inovação tecnológica.

A última vertente tem em Nonaka (ref. 024), Imai (ref. 021) e Takeuchi uma resposta positiva à procura americana da segunda vertente, ou vertente da simbiose. Nonaka e seus colegas apresentam empresas japonesas como a Honda e a Canon como exemplos de auto-renovação organizacional, ou seja, a resposta japonesa à excelência empresarial do Vencendo a Crise de Peters e Waterman. Um outro desenvolvimento japonês é a visão a partir da Organização Industrial, com base nas idéias de Oliver E. Williamson, que aponta a estrutura oligopolizada e de redes interligadas (com simbiose entre empresas) como verdadeira fonte do sucesso japonês, tendo em Aoki (ref. 001) seu representante mais conhecido em língua inglesa. Por último, vários outros autores têm feito esforços no sentido de inovação e renovação de modelos organizacionais, como Hioki (hólon, contingências, caos e pós-moderno), Kobayashi (organizing), Hashizumi etc.

A Administração Japonesa já possibilita e necessita uma história, coisa já feita em japonês, que descreva e explique sua evolução e que possa apontar seu futuro.

\section{REFERÊNCIAS BIBLIOGRÁFICAS}

Heraldo Vasconcellos - Bibliotecário, chefe do Serviço de Documentação da Biblioteca Karl A. Boedecker da EAESP/FGV.

\section{OBSERVAÇÕES}

A pesquisa foi realizada no acervo da Biblioteca da EAESP/FGV; as referências bibliográficas foram organizadas em ordem alfabética, dentro de cada ano de publicação (os anos aparecem em ordem cronológica decrescente). As referências precedidas de asterisco não estão disponíveis no acervo da Biblioteca.

\section{0}

001. AOKI, M. "Toward an economic model of the Japanese firm". Journal of Economic Literature, Nashville, 28(1):1-27, Mar. 1990.

002. BEAUCHAMP, M. "We're all rejuvenated (U.S. companies bought by Japanese)". Forbes,
New York, 145:124t, Mar. 19 1990.

003. BURSTEIN, Daniel. Yen: o Japão e seu novo império financeiro. São Paulo, Cultura, 1990. 384p.

004. HOLSTEIN, William J. et alii. "Hands across America: the rise of Mitsubishi". Business Week, New York, (3171):42-5, Sept. 241990.

005. "INNOVATION Japanesestyle". Strategic Direction, Zurich,(54): 8-11, Apr. 1990.

006. NEFF, Robert et alii. "Mighty Mitsubishi is on the move". Business Week, New York, (3171):38-41, Sept. 24 1990.

007. SCHWARTZ, Gilson. Japão de olhos abertos: evolução financeira e políticas econômicas na era moderna. São Paulo, Nobel, 1990. 153p.
008. THACKRAY, J. "In the shadow of the oriente (Japanese firms in U.S. fail to motivate their American managers)". Management Today, London:20, Feb. 1990.

\section{9}

009. BEDNARZIK, R.W. \& SHIELLS, C.R. "Labor market changes and adjustments: how to the U.S. and Japan compare". Monthly Labor Review, Washington, 112(2):31-42, Feb. 1989.

010. JAPANESE yearbook on business history. Tokyo, Japan Business History Institute, 1989. v.6

011. "MINTZBERG, H. "You meet the humblest managers on a Honda (book adaptation from Mintzberg on management)". Across the Board, 26.10-12, Oct. 1989.

012. MORTELLARO, J.S. "Business across a cultural void: Ja- pan's management imperialism". Business Marketing, Chicago, 74(2):62+, Feb. 1989.

013. "NEW business, new Japan (Japanese companies are getting more western)". The Economist, London, 312(7616):1112, Aug. 191989.

014. PARKER, P. "Five vital percent (Japanese and British management)". Management Today, London:112, Aug. 1989.

015. SCHRAGE, Michael. "A Japanese Giant Rethinks globalization: an interview with Yoshihisa Tabuchi". Harvard Business Review, Boston, 67(4):70-6, July/Aug. 1989.

016. WOLFEREN, Karel Van "The enigma of Japanese power". Fortune, Chicago, 119(10):98101, May 81989.

017. YOSHIDA, K. "Deming management philosophy: does it 
work in the U.S. as well as in Japan? (holistic thinking)". The Columbia Journal of World Business, New York, 24(3):10-17, Fall 1989.

018. *YOSHIMORI, M. As empresas japonesas. São Paulo, Martins Fontes, 1989.

\section{8}

019. ALLEN, L.A. "Working better with Japanese managers". Management Review, New York, 77(11):55-6, Nov. 1988.

020. HARPER, S.C. "Now that the dust has settled: learning from Japanese management". Business Horizons, Bloomington, 31(4):43-51, July/Aug. 1988.

021. IMAI, Masaaki. "Kaizen": a estratégia para o sucesso competitivo. São Paulo, Instituto de Movimentação e Armazenagem de Materiais, 1988. 236p.

022. JAPANESE Yearbook on Business History. Tokyo, Japan Business History Institute, 1988. v.5

023. "THE NAME of Japan's new business game". The Economist, London, 307(7545):67+, Apr. 91988.

024. NONAKA, I. "Creating organizational order out of chaos: self-renewal in Japanese firms". California Management Review, Berkeley, 30(3):57-73, Spring 1988.

025. RATTNER, Henrique. Impactos sociais da automação: 0 caso do Japão. São Paulo, Nobel, 1988. 122p.

\section{7}

026. AIDEN, Vernon R. "Who says you can't crack Japanese markets?" Harvard Business Review, Boston, 65(1):52-6, Jan./Feb. 1987.

027. BERGER, Michael. "The Japanese forge ahead to tackle their world problems". International Management, Lausanne, 42(3):43+, Mar. 1987.

028. DUNPHY, D. "Convergence/divergence: a temporal review on Japanese enterprise and its management". Academy of Management Review, Amherst, 12(3):445-59, July 1987
029. JAPANESE Yearbook on Business History. Japan Business History Institute, 1987. v.4

030. MATSUSHITA, Konosuke. Não vivemos somente pelo pão: um sistema de negócio, um princípio de gerenciamento. Tokyo, PHP, 1987. 157p.

031. MISAWA, M. "New Japanese-style management in a changing era". The Columbia Journal of World Business, New York, 22(4):9-17, Winter 1987.

032. "RATTNER, Henrique. Política industrial no Japão: tendências e perspectivas. São Paulo, Instituto de Pesquisas Econômicas da FEAVUSP, 1987. 34p.

033. ROHLEN, Thomas $P$. "Why Japanese education works?" Harvard Business Review, Boston, 65(5):42-3+, Sept./ Oct. 1987

034. UNO, Kimio. Japanese industrial performance. Amsterdam, North- Holland, 1987. 439p.

\section{6}

035. BAILLIE, A.S. "Subcontracting based on integrated standards: the Japanese approach". Journal of Purchasing and Materials Management, Albany, 22(1):17-22, Spring 1986

036. BEASLEY, W.G. (ed.) Modern Japan: aspects of history, literature and society. Bunkyo-ku, Tuttle, 1986. 296p.

037. HORNSTEIN, H.A. "Courageous management: America versus Japan". Personnel, New York, 63(7):22, July 1986.

038. JAPANESE Yearbook on Business History. Tokyo, Japan Business History Institute, 1986. v.3

039. *MEHTABDIN, K.R Comparative management. Lewiston, The Edwin Ellen Press, 1986.

040. MORITA, Akio. Made in Japan: Akio Morita e a Sony. São Paulo, Cultura, 1986. 334p.

041. PAPINOT, E. Historical and geographical dictionary of Japan. Sunkyo-ku, Tuttle, 1986. 842p.

042. THE TSUNETO YANO MEMORIAL SOCIETY. Nippon: a charted survey of Japan 1986/87. Tokyo, Kokusei-sha, 1986. 367p.

\section{5}

043. BUCKLEY, Roger. Japan today. Cambridge, Cambridge University, 1985. 139p.

044. *CÂMARA DE COMÉRCIO E INDÚSTRIA JAPONESA NO BRASIL. $O$ método japonês. São Paulo, Massao Ohno Ed., 1985. $103 p$.

045. CHIESL, Newell E. \& KNIGHT, Larry L. "Attitudes toward Japanese supply sources". Journal of Purchasing and Materials Management, Oradell, 21(2):2-6, Summer 1985.

046. CORTAZZI, H. "14 questions from Japan". Management Today, London:86-9, June 1985.

047. EXPERIENCING THE TWENTIETH CENTURY. Tokyo, University of Tokyo, 1985. 384p.

048. FREYSSENET, Michel \& HIRATA, Helena Sumiko. "Mudanças tecnológicas e participação dos trabalhadores: os círculos de controle de qualidade no Japão". Revista de Administração de Empresas, São Paulo, 25(3):5-21, jul./set. 1985.

049. HANN, P. "Umeo Oyama: this corporate doctor with homespun remedies is no quack". International Management (Europe Edition), Maidenhead 40(5):53+, May 1985.

050. HUPPERTS, I. "How western-style corporate identity programmes aid Japanese firms". International Management (Europe Edition), Maidenhead, 40(3):66+, Mar. 1985.

051. *JAEGER, A.M. \& BALIGA, B.R. "Control systems and strategic adaptation: lessons from the Japanese experience". Strategic Management Journal, 6:115-34, Apr./June 1985.

052. KOTLER, Philip. The new competition. Englewood Cliffs, Prentice-Hall, 1985. 292p.

053. LAUENSTEIN, M.C. "International business strategy: strategic planning in Japan". Journal of Business Strategy, Boston, 6(2):78-84, Fall 1985.

054. LEWIS JR., James. Excellent organizations: now to develop \& manage then using the- ory Z. New York, Wilkerson, 1985. 307p.

055. MANASIAN, D. "Where Japan's biggest are better". Management Today, London:72-5+, July 1985

056. *MISUMI, J. \& PETERSON, M.F. "The performancemaintenance (PM) theory of leadership: review of a japanese research program". Administrative Science Quarterly, Ithaca, 30:198-223, June 1985.

057. MORGAN, J.P. "The facts about just-in-time, Japan, and Japanese business (J.P. Flanagan)". Purchasing, Boston, 99(12):4350, Dec. 191985.

058. NAKANE, C. Japanese society. Bunkyo-ku, Tuttle, 1985. 162p.

059. NEGANDHI, A.R. et alii. "The management practices of Japanese subsidiaries overseas". California Management Review, Berkeley, 27(4):93-105, Summer 1985.

060. "NOW from Japan: intravenous management (bood type personality analysis)". International Management (Europe Edition), Maidenhead, $40(7): 7+$, July 1985.

061. OHMAE, Konichi. $O$ estrategista em ação: a arte japonesa de negociar. São Paulo, Pioneira, 1985. 277p.

062. SATOSHI, Kamata. Japão: a outra face do milagre. São Paulo, Brasiliense, 1985. 169p.

063. SUG0, Alberto Issao. 0 mito do sistema administrativo japonês. Uma tentativa de interpretação alternativa do sistema administrativo japonês como fenômeno social. São Paulo, EAESP/FGV, 1985. 200p.

064. SUZAWA, S. "How the Japanese achieve excellence". Training and Development Journal, Alexandria, 39(5):110-12+, May 1985

065. THUROW, Lester C. The management challenge: japanese views. Cambridge, MIT, 1985. $237 \mathrm{p}$.

066. UNIVERSIDADE FEDERAL DE SÃO CARLOS. Departamento de Engenharia da Produ- 
ção. Japão: 0 debate sobre a importação de um modelo. São Carlos, 1985. 129p.

\section{4}

067. "CHERRY blosson in Pittsburgh (survey into American and Japanese management)". The Economist, London, 293:77, Nov. 101984

068. ECONOMIC SURVEY OF JAPAN: 1983/1984. Tokyo, Economic Planning Agency, 1984. $206 p$.

069. ETO, Hajime \& MATSUI, Konomu. (eds.) $R \& D$ management system in Japanese industry. Amsterdam, North-Holland, 1984. 331p.

070. FARIA, José Henrique. "Círculos de controle de qualidade: a estratégia recente da gestão capitalista de controle e modificação do processo técnico do trabalho". Revista de Administração, São Paulo, 19(3):9-16, jul./set. 1984.

071. GAINO, Luciano S. "Fatores da produtividade japonesa". Revista de Administração de Empresas, São Paulo, 24(2):41-2, abr./jun. 1984.

072. GIBNEY, Frank. Japan: the fragile superpower. Bunkyoku, Tuttle, 1984. 378p.

073. “THE HERETIC Who's hero to Japanese business (K. Inamori)". Business Week, Highstown:99-100+, Apr. 161984.

074. IIDA, Itiro. Pequena e média empresa no Japão. São Paulo, Brasiliense; Brasília, CN$\mathrm{Pq}$, 1984. 279p.

075. "JAPANESE management: setting the record straight". Management Review, New York, 73(2):55, Mar. 1984.

076. JETRO. Introduccion a la economia y comercio exterior de Japon. São Paulo, 1984. $68 \mathrm{p}$.

077. KEYS, J.B. \& MILLER, T.R. "The Japanese management theory jungle". Academy of Management Review, Amherst, Y(2):342-53, Apr. 1984.

078. KONO, Toyohiro. Strategy and structure of Japanese enterprises. London, MacMillan, 1984. 352p.

079. LEBRA, Takie Sugiyama. Japanese patterns of behavior. Honolulu, University of Hawaii, 1984. 295p.

080. LIFSON, T.B. "Adaptation: a key to organizational health". Research Management, Lancaster, 27(4):37-40, July/Aug. 1984.

081. McMILLAN, Charles J. The Japanese Industrial System. Berlin, Walter de Gruyter, 1984. $356 \mathrm{p}$.

082. MAIN, J. "The trouble with managing Japanese-style". Fortune, Chicago, 109(7):10-14, Apr. 21984.

083. NEFF, R. "Kenichi Ohmae: the impresario of Japanese management consulting". International Management (Europe Edition), Maidenhead, 39(11):735, Nov. 1984.

084. ODIORNE, George S. "The trouble with Japanese management systems". Business Horizons, Indiana, 27(4):17-23, July/Aug. 1984.

085. OUCHI, William G. "Political and economic teamwork: the development of the micro eletronics industry of Japanese". California Management Review, Berkeley, 26(4):8-34, Summer 1984.

086. PHALON, R. "Hell camp (management training camp; Kanrisha)". Forbes, New York, 133(14):56-8, June 181984.

087. REISCHAUSER, Edwin 0. Japan: tradition \& transformation. Bunkyo-ku, Tuttle, 1984. $347 p$.

088. SCHONBERGER, Richard J. Técnicas industriais japonesas: nove lições ocultas sobre simplicidade. São Paulo, Pioneira, 1984. 309p.

089. SETHI, S.P. et alii. "The decline of the Japanese system of management". California Management Review, Berkeley, 26(4):35-45, Summer 1984.

090. WEISS, Andrew. "Simple truths of Japanese manufacturing". Harvard Business Review, Boston, 62(4):119-25, July/Aug. 1984.

\section{3}

091. ALLEN, G.C. A Economia Japonesa. Rio de Janeiro, Zahar, 1983. 245p.

092. BICKERSTAFFE, G. “The mixed scorecard of Japanese management abroad". International Management (Europe Edition) Maidenhead, $38(7): 12-6$, July 1983.

093. BOYER, Edward. "How Japan manages declining industries". Fortune, Chicago, 107(1):34-9, Jan. 10, 1983

094. DILLON, L.S. "Adopting Japanese management: some cultural stumbling blocks". Personnel, Saranac Lake, 60(4):737, July/Aug. 1983.

095. "THE HIDDEN Japanese weakness: manufacturing abroad (editorial)". International Management (Europe Edition), Maidenhead, 38(7):2, July 1983.

096. *HIRATA, Helena Sumiko. "Receitas japonesas, realidade brasileira". Novos Estudos Cebrap, São Paulo, 2(2):61-5, Jul. 1983.

097. LEBRA, Takie S. \& LEBRA, William P. (ed.) Japanese culture and behavior: selected readings. 4a. ed. Honolulu, University of Hawaii, 1983. 459p.

098. LICKER, P.S. "On beyond $Z$ : Japanese management style might Seuss us just fine". Journal of Systems Management, Cleveland, 34(10): 10-13, 0ct. 1983.

099. REID, R.A. \& SMITH, H.L. "Concepts underlying Japanese management (implications for purchasing and materials managers)". Journal of Purchasing and Materials Management, Albany, 19(4):14-20, Winter 1983.

100. *ROBBINS, Stephen P. "The theory $Z$ organization from a power control perspective". California Management Review, Berkeley, 25(2): 67-75, Dec. 1983.

101. ROHLEN, T.P. "Sponsor hip of cultural continuity in Japan: a company training program". In: LEBRA, Takie S. \& LEBRA, W.P. Japanese culture and behavior: selected readings. Honolulu, University of Hawaii, 1983. 459p.
102. ZIERDEN, W.E. "The real difference between the Japanese and U.S. managers (attitudinal differences)". Advanced Management Journal, Cincinatti, 48(4):59-62, Autumn 1983.

\section{2}

103. BOCKER, H.J. \& OVERGAARD, H.O. "Japanese quality circles: a managerial response to the productivity problem". Management International Review, Wiesbadeu, 22(2):13-19, 1982.

104. *"C.C.Q.: o que está por trás disso?" Tribuna Metalürgica(2): jan. 1982. (supl.)

105. KONO, T. "Japanese management philosophy: can it be exported?" Long Range Planning, Oxford, 15(3):90-102, June 1982.

106. *MARSLAND, S. \& BEER, $M$. "The evolution of Japanese management: lessons for U.S. managers". Organizational Dynamics, 11:49-67, Winter 1982.

107. NAKAMURA, Takafusa. The postwar japanese economy. Tokyo, University of Tokyo, 1982. $277 p$.

108. "NAKAOKA, Tetsuro. "The role of domestic technical innovation in foreign technology transfer; the case of Japanese cotton textile industry". Osaka City University Economic Review, 18:45-62, 1982.

109. OUCHI, William G. Teoria $Z$ : como as empresas podem enfrentar 0 desafio japonês. $2^{\mathrm{a}}$ ed. São Paulo, Fundo Educativo Brasileiro, 1982. 293p

110. *OZAWA, T. "Japanese chic". Across the Board, 19:6-13, Oct. 1982.

111. PARK, J.C. "Need for managerial changes in U.S. corporations". Managerial Planning, 0xford, 30(6):26-30, May/June 1982.

112. PASCALE, Richard Tanner \& ATHOS, Anthony G. As artes gerenciais japonesas. Rio de Janeiro, Record, 1982. 247p.

113. SULLIVAN, J. \& PETERSON, R.B. "Factors associated with trust in Japanese-American joint ventures". Management $/ n$ - 
ternational Review, Wiesbadeu, 22(2):30-40, 1982.

\section{1}

114. "AMERICAN myths vs the real reasons for Japan' s success". Management Review, New York, 70(6):55-6, June 1981.

115. ARBOSE, J.R. "Is Japan cornering the market on product quality?" International Management, Maidenhead, 36(1):22-5, Jan. 1981

116. BOWEN, W. "Lessons from behind the Kimono". Fortune, Chicago, 103(12):247-8+, June 151981.

117. DRUCKER, P.F. "Behind Japan' s success". Harvard Business Review, Boston, 59(1):8390, Jan./Feb. 1981

118. HATVANY, N. \& PUICK, V. "An integrated management system: lessons from the Japanese experience". Academy of Management Review, Amherst, 6(3):469-80, July 1981.

119. HAYES, R.H. "Why Japanese factories work". Harvard Business Review, Boston, 59(4):56-66, Jul. 1981.

120. "HOW the Japanese manage in the US (panel discussion)". Fortune, Chicago, 103(12):97-8+, June 151981.

121. *KAGONO, $T$. et alii. "Mechanistic vs. organic management systems: a comparative study of adaptative patterns of U.S. and Japanese firms". Annals of the School of Business Administration, 25:115-45, 1981.

122. LIM, H. "Japanese management: a skill profile". Training and Development Journal, Alexandria, 35(10):18-21, 0ct. 1981.

123. LINCOLN, J.R. et alii. "Cultural orientations and individual reactions to organizations: a study of employees of Japaneseowned firms". Administrative Science Quarterly, Ithaca, 26:93115, 1981.

124. MASAOKA, Katsuji. $A d-$ ministração japonesa: sua adaptação no Brasil. São Paulo, EAESP/FGV, 1981. 142p.

125. "NAKAOKA, Tetsuro.
"Production management in Japan before the period of high economic growth". Osaka City University Review, 17:7-24, 1981.

126. REHDER, R.R. “Japan's synergistic society: how it works and its implications for the United States". Management Review, New York, 70(10):64-6, Oct. 1981.

127. "What American and Japanese managers are learning from each other". Business Horizons, Bloomington, 24(2):63-70, Mar./Apr. 1981.

128. RICHARDSON, Bradley M. (ed.) Business and society in Japan: fundamentals for businessmen. New York, Praeger, 1981. 334p.

129. SCHEIN, Edgard $H$. "SMR FORUM does Japanese management style have a message for American managers?" Sloan Management Review, Cambridge, 23(1):55-68, Fall 1981.

130. TAKAMYA, Makoto. "Japanese multinationals in Europa: international operation and public policy implications". Columbia Journal of World Business, New York, 16(2):5-17, Summer 1981.

131. WHEELWRIGHT, S.C. "Japan-where operations really are strategic". Harvard Business Review, Boston, 59(4):7-74, July/Aug. 1981.

\section{0}

132. COLE, R.E. “Learning from the Japanese: prospects and pitfalls". Management Review, New York, 69(9):22-8+, Sept. 1980.

133. ISHIKAWA, Akihiro. "Des lip Nippon”. Autogestions, Paris, 13(1):95-110, Jan. 1980.

134. "Modèles et expériences autogestionnaires au Japon". Autogestions, Paris, 13(1):79-93, 1980.

135. OZAMA, Terumoto. "Japan's industrial groups". MSU Business Topics, East Lansing, 28(4):33-41, Autumn 1980.

136. * "Japanese world of work: an interpretative survey". MSU Business Topics, East Lansing, 28(2):45-55, Autumn 1980.
137. SAITO, Hiroshi. "A relação entre a cultura e os padrões de comportamento de um lado e a ciência e a tecnologia de outro". In: CÂMARA DE COMÉRCIO E INDÚSTRIA JAPONESA DO BRASIL. I Seminário "A cultura japonesa e sua influência na atividade empresarial". São Paulo, ago. 1980. p.29-32.

138. SEMINÁRIO. CÂMARA DO COMÉRCIO E INDÚSTRIA JAPONESA DO BRASIL, $1^{0}$ Seminário "A cultura japonesa e sua influência na atividade empresarial". São Paulo, ago. 1980. 36p.

139. *SUZUKI, Y. "The strategy and structure of top $100 \mathrm{Ja}$ panese industrial enterprises 1950-1970". Strategic Management Journal, 1: 265-91, 1980.

140. TSUKAMOTO, $Y$. "O processo decisório empresarial no Japão". Revista de Administração de Empresas, São Paulo, 20(4):73-7, out./dez. 1980.

141. "A U.S. turn to native talent in Japan". Business Week, Hightstown:56+, Dec. 81980.

142. WOOD, R.C. “Japan's multitier wage system". Forbes, New York, 126(4):53+, Aug. 18 1980.

\section{9}

143. ATSUMI, Reiko Tsukiai "Obligatory personal relationship of Japanese white-collar company employees". Human Organization, Boulder, 38(1):63-70, Spring 1979.

144. CRAIG, Albert M. (ed.) Japan: a comparative view. Princeton, Princeton University, 1979. $437 p$.

145. DONALDSON, Lex. "Regaining control at Nippont". Journal of General Management, Oxford, 4(4):14-31, Summer 1979 .

146. *ISHIDA, Hideo. O sistema administrativo japonês no exterior. São Paulo, EAESP/FGV/JETRO, 1979.

147. SUMIYA, Mikio, (ed.) An outline of Japanese economic history 1603-1940. Tokyo, University of Tokyo, 1979. 372p.

\section{8}

148. HAYASHI, Kichiro. “Japanese management of multinational operations". Management $/ n-$ ternational Review, Wiesbadeu, 18(4): 47-57, Out 1978.

149. LINCOLN, J.R. "Cultural effects on organizational structures: the case of Japanese firms in the United States". American Sociological Review, 43:829-47, 1978.

150. PASCALE, R.T. "Communication and decision making across cultures: Japanese and American comparisons". Administrative Science Quarterly, Ithaca, 23(1):91-110, Mar. 1978.

\section{7}

151. HAZAMA, Hiroshi. "Formation of the management system in Mejii Japan: personnel management in large corporations". Developing Economies, Tokyo, 15(4):402-19, Dec. 1977.

152. HILL, R. "Japanese fibres firm restructures to restore profits". International Management, Maidenhead, 32(4):41-3, Apr. 1977.

153. JOHNSON, R.T. "Success and failure of Japanese subsidiaries in America". Columbia Journal of World Business, New York, 12(1):30-7, Mar. 1977

154. MARSH, R.M \& MANNARI, H. "Organizational commitment and turnover: a prediction study". Administrative Science Quarterly, Ithaca, 22(1):57-75, Mar. 1977

155. *YOSHIDA, M. "An observation on the foundation of $\mathrm{Hi}$ tachi Seisakusho-prehistory of the Hitachi trust". Keio Business Review, Tokyo, (14):97-119, 1977.

\section{6}

156. ${ }^{\star} A Z U M I, K . \&$ McMILLAN, C.J. "Worker sentiment in the Japanese factory: its organizational determinants". In: AUS TIN. (Ed.) Japan: the paradox of progress. New Haven, Yale University Press, 1976. p.215-29.

157. TRACY, P. \& AZUMI, K. "Determinants of administrative control: a test of theory with Ja- 
panese factories". American Sociological Review, Washington, 41(1):80-94, Feb. 1976.

158. *YAMAMOTO, H. "Productivity bargaining and the change of labour management relations in the Japanese foreign going shipping; 1965-1975". Kobe Economic \& Business Review, Kobe, (22):17-25, 1976.

\section{5}

159. *AZUMI, K. \& MCMILLAN, C.J. "Culture and organization structure: a comparison of Japanese and British organizations". International Studies of Management and Organization, 5(1):35-47, 1975.

160. *ENGLAND, George William. The manager and his values: an international perspective from the United States, Japan, Korea, India and Australia. Cambridge, Ballinger, 1975.

161. *FODELLA, Gianni. (ed.) Social structures and economic dynamics in Japan up to 1980. Milano, Università Bocconi, 1975.

162. MIZUNO, Hajime. "A economia japonesa após a crise do petróleo e as implicações nas suas relações com a América Latina". Revista de Administração de Empresas, São Paulo, 15(5):40-51, set./out. 1975.

163. VOGEL, Ezna F. (ed.) Modern Japanese organization and decision making. Berkeley, University of California, 1975. 340p.

\section{4}

164. *NOGUTI, T. "The formation of the Japanese Zaibatsu the political merchant in the original accumulation of capital". Keio Business Review, (16):169-87, 1974.

165. "YANAGIHARA, N. "The strategy and structure of Japanese industrial corporations". KSU Economic and Business Review: 24-25, 1974.

\section{3}

166. *ABEGGLEN, J.C. Management and worker: the Japanese solution. Tokyo, Kodansha International, 1973.
167. DORE, Ronald. British factore-Japanese factory: the origins of national diversity in industrial relations. Berkeley, University of California, 1973. 432p.

168. HALLIDAY, Jon \& Mc CORMACK, Gavan. Japanese imperialism today: co-prosperity in greater East Asia. New York, Monthly Review, 1973. 279p.

169. *KELLEY, L. \& REESER, C. "The persistence of culture as a determinant of differentiated attitudes on the part of American managers of Japanese ancestry". Academy of Management Journal, 16:67-76, 1973.

170. LIVINGSTON, Jon. (ed.) The Japan reader. New York, Pantheon, 1973. 2v.

171. "MARCH, R.M. \& MANNARI, H. “Japanese worker's responses to mechanization and automation". Human Organization, Boulder, 32(1): 85-93, 1973.

172. *PARKANSKII, A. "Export of American methods of management to Japan". Japanese Economic Studies, 2(2):65-76, 1973.

173. ROHLEN, Ṫhomas P. "Spiritual education in Japanese bank". American Anthropologist, Washington, 75(5):1542-62, Oct. 1973.

\section{2}

174. BENEDICT, Ruth. $0 \mathrm{cri-}$ sântemo e a espada: padrões da cultura. São Paulo, Perspectiva 1972. 277p.

175. KOSAKA, Masataka. 100 million Japanese: the postwar experience. Tokyo, Kodansha, 1972. $282 p$.

176. "MARSH, R.M. \& MANNARI, H. "A new look at lifetime commitment in Japanese industry". Economic Development and Cultural Change, Chicago, 20.611-30, 1972.

177. VARLEY, H. Paul. A syllabus of Japanese civilization. $2^{\mathrm{a}}$.ed. New York, Columbia University, 1972. 98p.

\section{1}

178. KAHN, Herman. The emerging japanese superstate: challenge and response. Englewood Cliffs, Prentice-Hall, 1971. 274p.
179. YOSHINO, M.Y. The Japanese marketing system: adaptions and innovations. Cambridge, MIT, 1971. 319p.

\section{0}

180. DERRUAU, Max. O Japão. São Paulo, Difusão Européia, 1970. 282p.

181. *ENGLAND, G.W. \& KOI$K E, R$. "Personal value systems of Japanese managers". Journal of Cross-Cultural Psychology, 1:21-40, 1970.

182. *EVANS, R. "Evolution of the Japanese system of employer-employee relations, 18681945". Business History Review, 44(1):110-25, 1970.

\section{9}

183. IMAI, M. "Shukko, Jomukai, Ringi - the ingredients of executive selection in Japan". Personnel, 46(4):20-30, Jul. 1969.

\section{8}

184. BEASLEY, W.G. História moderna del Japón. Buenos Aires, SUR, 1968. 337p.

185. *KARSH, B. \& COLE, R.E. "Industrialization and the convergence hypothesis: some aspects of contemporary Japan". Journal of Social Issues, 24(4):45-64, 1968.

186. YOSHINO, M.Y. Japan's managerial system: tradition and innovation. Cambridge, Institute Technology, 1968. 292p.

\section{7}

187. BALLON, Robert J. (ed.) Doing business in Japan. Tokio, Sophia University, 1967. 215p.

188. MARSHALL, Byron K. Capitalism and nationalism in prewar Japan: the ideology of the business elite, 1868-1941. Stanford, Stanford University, 1967. 163p.

\section{6}

189. *BRONFERNBRENNER, Martin. "'Excessive competition' in Japanese business". Monumenta Nipponica, 21(1-2):11424, 1966.
190. *BROWN, William. “Japanese management: the cultural background". Monumenta Nipponica, 21(1-2):47-60, 1966.

191. "CHANDLER, M.K. "Management rights: made in Japan". Columbia Journal of World Business, New York, 1(1):131-40, Jan. 1966.

\section{4}

192. YAMASHIRO, José. Pequena história do Japão. ${ }^{2}$. ed. São Paulo, Herder, 1964. 198p.

\section{2}

193. *MIZUNO, T. "Labor shortage in Japan-its new phase and effect on the small business". Kobe University Economic Review, (8):109-18, 1962.

194. *TAIRA, K. "Characteristics of Japanese labor markets". Economic Development and Cultural Change, Chicago, 10.150-68, 1962.

\section{0}

195. *FUJII, Shiguero. "Functions of small-and-medium-scale exporting firms". Kobe University Economic Review, (6):1-16, 1960.

\section{9}

196. *MIZUNO, T. "Problems of small and medium industries in Japan". Kobe University Economic Review, (5):75-88, 1959.

\section{8}

197. *ABEGGLEN, J.C. The Japanese factory: aspects of its social organization. Glencoe, Free Press, 1958.

198. TSURU, Shigeto. Essays on Japanese economy. Tokyo, Kinokuniya, 1958. 241p.

\section{7}

199. ABEGGLEN, J.C. "Subordination and autonomy attitudes of Japanese workers". American Journal of Sociology, 63:181-9, 1957

\section{6}

200. *ISHINO, Iwao. "Motivational factors in Japanese labor supply organization". Human Organization, Boulder, 15(2):12-17, Summer 1956. 